\title{
ATENCIÓN OBSTÉTRICA A MUJERES DIAGNOSTICADAS DE ESQUIZOFRENIA
}

\section{ESTEFANÍA MUÑOZ ALBA}

Matrona. Unidad de Cuidados de Alto Riesgo Obstétrico y Paritorio. Hospital Clínico San Carlos. Madrid.

\section{RESUMEN}

Objetivos: establecer las implicaciones esenciales del trastorno del espectro de la esquizofrenia en las mujeres que atraviesan el período de embarazo y posparto desde el punto de vista obstétrico; poner de relieve aspectos fundamentales de la atención del recién nacido de madre con diagnóstico de esquizofrenia, tras el parto y durante la lactancia materna.

Metodología: se ha realizado una revisión bibliográfica mediante el empleo de diversas bases de datos: PubMed, SAGE, ScienceDirect, Scielo, Web of Science y PsycINFO. Descriptores utilizados: esquizofrenia, atención prenatal, atención posnatal, fármacos antipsicóticos, lactancia materna, rehabilitación psiquiátrica, trastornos psicóticos.

Resultados: en las mujeres embarazadas diagnosticadas de esquizofrenia, es imprescindible la valoración de la relación beneficio-riesgo para el tratamiento antipsicótico. Los resultados obstétricos son consecuencia del estilo de vida, la sintomatología y el tratamiento antipsicótico, así como de otras afecciones médicas concomitantes. Es imprescindible la estrecha observación del recién nacido tras el parto y durante la lactancia materna, así como el seguimiento óptimo de la madre en el puerperio.

Conclusiones: las mujeres diagnosticadas de esquizofrenia y en proceso de gestación presentan necesidades en diversas esferas de su vida, precisando atención sociosanitaria a lo largo de toda su vida. En lo referente al tratamiento médico, se emplean antipsicóticos, previa información y consenso con la paciente y su red de apoyo familiar. Después del parto y durante la lactancia materna, los recién nacidos son más vulnerables, dada su exposición al tratamiento antipsicótico, y deben ser estrechamente vigilados.

Palabras clave: esquizofrenia, atención prenatal, atención posnatal, fármacos antipsicóticos, lactancia materna.

Correspondencia: Estefanía Muñoz Alba.

Email:esmual@live.com 


\section{INTRODUCCIÓN}

En el período del embarazo, acontecen profundos cambios que atañen a todos los ámbitos del ser humano. Se producen numerosas modificaciones fisiológicas en el cuerpo de la futura madre para favorecer el desarrollo embrionario y fetal. A nivel psicológico, las nuevas exigencias acaecidas en torno a la maternidad implican la puesta en marcha de recursos cognitivos, emocionales y conductuales, que permitan la adaptación frente al nuevo contexto familiar. A nivel social, fluyen nuevas expectativas sobre el rol materno y el cuidado y bienestar del recién nacido. No obstante, el diagnóstico de esquizofrenia empaña estos aspectos, pues se trata de un trastorno mental grave con gran impacto en la vida de la mujer y en un ambiente en el que aún predomina el estigma social.

La esquizofrenia es un tipo de trastorno psicótico. Según la quinta edición del Manual diagnóstico y estadístico de los trastornos mentales ${ }^{1}$, para el diagnóstico de esquizofrenia, se requiere, entre otros criterios, que la persona muestre, al menos, dos síntomas de los que se listan a continuación, debiendo presentar, como mínimo, uno de los tres primeros referidos: delirios (creencias fijas de las percepciones o experiencias), alucinaciones (suelen ocurrir en cualquier modalidad sensorial, pero las más comunes son las alucinaciones auditivas), pensamiento desorganizado, comportamiento desorganizado y síntomas negativos (abulia, disminución de la expresión emocional, etc.).

Además de esta sintomatología, se ponen de manifiesto deficiencias cognitivas en diversas áreas, afectando todo ello a la capacidad de vivir de forma independiente y al funcionamiento cotidiano ${ }^{2}$. En este

\section{Es frecuente que estas mujeres no realicen los cuidados prenatales necesarios para un correcto seguimiento de la gestación.}

contexto, tanto el bienestar materno y del recién nacido como el vínculo entre ambos pueden verse comprometidos.

\section{DESARROLLO Y DISCUSIÓN}

\section{Metodología}

Se ha realizado una revisión bibliográfica, para la que se han empleado diversas bases de datos: PubMed, SAGE, ScienceDirect, Scielo, Web of Science y PsycINFO. Los descriptores utilizados fueron: esquizofrenia, atención prenatal, atención posnatal, fármacos antipsicóticos, lactancia materna, rehabilitación psiquiátrica, trastornos psicóticos. Se obtuvieron un total de 58 registros, de los que se seleccionaron 31 , que contienen los aspectos formales esenciales de la presente revisión.

\section{Resultados}

\section{Estilo de vida y salud}

Diversos factores del estilo de vida de las personas afectadas de esquizofrenia contribuyen a un empeoramiento de su salud ${ }^{3}$.

En comparación con la población general, en estas personas, es más frecuente la presencia de factores de riesgo que favorecen el síndrome metabólico: una dieta inadecuada, el sedentarismo, el hábito tabáquico y la obesidad ${ }^{4}$. Igualmente, se destaca una especial comorbilidad con la diabetes de tipo 2 , debido a la influencia directa e indirecta de dichos factores en su desarrollo, a lo que se suman alteraciones metabólicas propias de algunos fármacos antipsicóticos que contribuyen a la hiperglucemia, la hiperlipidemia y el aumento de peso ${ }^{4}$. Incluso ciertos determinantes sociales, como la pobreza, generan altos y prolongados niveles de estrés, que terminan por perturbar el sistema inmunitario. Por último, cabe reseñar la propia vulnerabilidad genética o neurobiológica al desarrollo de la diabetes y de otras 
enfermedades ${ }^{4,5}$. También se ha descrito en esta población una mayor prevalencia de alteraciones derivadas del consumo de sustancias, entre las que figuran la nicotina, el alcohol, el cannabis y la cocaína ${ }^{6}$. Además, es frecuente que presenten comorbilidad con síntomas depresivos y ansiosos ${ }^{7}$. La depresión es uno de los principales factores de riesgo para cometer suicidio en personas con esquizofrenia. Otros factores influyentes son: el historial de intento de suicido, el abuso de drogas, la propia sintomatología de la esquizofrenia, la falta de adherencia terapéutica y la mendicidad. Como factor protector, se enfatiza el adecuado tratamiento de la esquizofrenia y de los problemas asociados ${ }^{8}$.

Asimismo, se han identificado diversos déficits cognitivos en diferentes áreas de habilidad: velocidad psicomotriz, atención, memoria y funciones ejecutivas (planificación, resolución de problemas, organización, toma de decisiones, etc.). Todo ello está en la base de sus carencias en cuanto a relaciones sociales, el aprendizaje en programas de rehabilitación, y el desempeño de un trabajo cualificado y de las actividades de la vida diaria9 .

En lo referente a las relaciones interpersonales, muestran un mayor aislamiento $\mathrm{y}$, cuando interaccionan con otras personas, tienen dificultades para mantener una conversación apropiada, expresar sus necesidades y sentimientos, alcanzar metas sociales o desarrollar relaciones cercanas ${ }^{9}$. También es más frecuente la separación y el divorcio que en la población general ${ }^{10}$.

A nivel social, la mendicidad es probablemente la secuela más visible de los trastornos psicóticos, dados los múltiples factores que afectan a la calidad de vida ${ }^{10}$.

\section{Tratamiento antipsicótico}

Si bien la etiología de la esquizofrenia es multifactorial e involucra diversos mecanismos neurobiológicos, es necesario señalar la disfunción del sistema dopaminérgico y el papel que desempeñan los fármacos antipsicóticos en la regulación de los sínto- mas de la psicosis, así como su seguridad en la etapa gestacional, pues todos los antipsicóticos atraviesan la placenta, suponiendo un riesgo para el desarrollo fetal y el estado neonatal ${ }^{11}$.

Se distinguen dos grupos de antipsicóticos ${ }^{12}$ : anti psicóticos típicos y antipsicóticos atípicos.

Los antipsicóticos típicos, o antipsicóticos de primera generación, ejercen su acción mediante el bloqueo de los receptores dopaminérgicos $\left(D_{2}\right)$ de la vía dopaminérgica mesolímbica, reduciendo la hiperactividad de esta vía. A nivel clínico, esto se traduce en una disminución de los síntomas positivos de la psicosis (delirios, alucinaciones, discurso incoherente, etc. ${ }^{12}$. No obstante, el bloqueo no se produce exclusivamente en la vía mencionada, sino que el fármaco se distribuye por otras vías neurobiológicas, produciendo diversos efectos colaterales no deseados, entre ellos: síntomas extrapiramidales, sedación, hiperprolactinemia, hipotensión postural, efectos anticolinérgicos (visión borrosa, boca seca, retención urinaria), alteraciones cardíacas y síndrome neuroléptico maligno ${ }^{12,13}$. De este grupo, los fármacos antipsicóticos más evaluados durante el embarazo son las fenotiazinas (clorpromazina) y el haloperidol ${ }^{14}$. En lo concerniente a efectos teratógenos, no se ha evidenciado un patrón específico de anormalidades, y las tasas detectadas de malformaciones se encuentran por debajo del $3 \%$ de la población general. Su uso durante el tercer trimestre se ha asociado a un mayor riesgo de síndrome de abstinencia neonatal y síntomas extrapiramidales en el neonato ${ }^{15}$.

Los antipsicóticos atípicos o de segunda generación son antagonistas serotoninérgicos-dopaminérgicos. En general, mejoran los síntomas positivos y negativos, existiendo, por otro lado, cierta heterogeneidad en cuanto a efectos adversos ${ }^{12}$. Groso modo, causan menos síntomas extrapiramidales y producen escaso o nulo aumento en la secreción de prolactina (a excepción de la risperidona y la amisulprida). Algunos de ellos (olanzapina y clozapina) están asociados a un mayor riesgo de incremento de peso, alteraciones en la glucemia y diabetes ${ }^{12,13,15,16}$, Io 
que repercute más directamente en potenciales complicaciones obstétricas. La evidencia disponible sobre la seguridad de los antipsicóticos de segunda generación es limitada, si bien, no parece existir un riesgo teratógeno consistente con la administración de clozapina, olanzapina, risperidona y quetiapina ${ }^{15,17}$. En lo referente a los efectos en el neonato, se ha documentado una mayor incidencia de recién nacidos grandes para la edad gestacional ${ }^{15}$.

Seguidamente, se exponen diversas consideraciones de interés sobre el tratamiento farmacológico con antipsicóticos en la mujer embarazada, desarrolladas por la agencia NICE ${ }^{18}$ :

- Optar por el fármaco con menor riesgo para la mujer, el feto y el recién nacido, considerando la respuesta previa de la mujer a la medicación.

- Usar la mínima dosis eficaz.

- Usar un único fármaco en la medida de lo posible (la polifarmacia aumenta el riesgo de teratogenicidad).

- En el caso de que la mujer quiera dejar de tomar la medicación, valorar con ella (y su familia) riesgos y beneficios, y plantear la posibilidad de cambiar de fármaco, así como de realizar intervenciones psicológicas complementarias.

- Nunca suspender de forma brusca el tratamiento con antipsicóticos.

\section{Embarazo, parto y puerperio}

La psicosis puede contribuir a la negación del embarazo, a malinterpretar los cambios corporales propios de la gestación o a una falta de reconocimiento del inicio del parto ${ }^{10}$. Es frecuente que estas mujeres no realicen los cuidados prenatales necesarios para un correcto seguimiento de la gestación ${ }^{19}$. Además, la enfermedad psicótica puede interferir en la habilidad parental e, incluso, causar la pérdida de la custodia de los hijos ${ }^{20}$. A este respecto, es básico fomentar la adherencia terapéutica, al tiempo que dotar de habilidades sociales, ya que este tipo de entrenamiento supone un factor de protec-
Es básico fomentar la adherencia terapéutica, al tiempo que dotar de habilidades sociales.

ción ante el estrés, ayuda a estabilizar los síntomas, a hacer a las personas más resistentes y adheridas a su tratamiento y a mejorar las posibilidades de integración social ${ }^{21}$. El tratamiento de la esquizofrenia debe contemplar de forma integrada aspectos psicofarmacológicos, intervenciones psicosociales y rehabilitación social, y una buena coordinación interinstitucional con la colaboración de profesionales sanitarios, de servicios sociales y también del sistema de empleo, educativo, judicial, etc. ${ }^{22}$.

En esta población, existe un mayor riesgo de diabetes gestacional, preeclampsia ${ }^{23,24}$ y enfermedad tromboembólica ${ }^{24}$, lo que viene condicionado por factores de riesgo propios de su estilo de vida (altos niveles de tabaquismo, sedentarismo, dieta inadecuada), el consumo de antipsicóticos atípicos, que favorecen el síndrome metabólico, el pobre cuidado antenatal y las enfermedades preexistentes al momento del embarazo ${ }^{23,24}$.

No se han evidenciado resultados adversos en el parto debido al uso de antipsicóticos ${ }^{15}$. Según los estudios, en esta población se ha descrito un mayor riesgo de parto pretérmino y de recién nacidos pequeños para la edad gestacional15,24, debido probablemente a la confluencia de diversos factores (comorbilidad, déficit de autocuidados, dificultades de acceso a los servicios de salud, etc.). Por otro lado, en las mujeres expuestas a antipsicóticos de segunda generación, se ha observado una mayor incidencia de recién nacidos grandes para la edad gestacional, dados los efectos metabólicos de algunos de estos fármacos, que favorecen el aumento de peso y el desarroIlo de diabetes en la madre ${ }^{15}$. Es fundamental realizar las pruebas de cribado de la diabetes y, en caso de presentar diabetes gestacional, emprender acciones para mantener la normoglucemia, dadas las complicaciones perinatales a las que va asociada ${ }^{25}$. 
En el neonato, hay un mayor riesgo de síndrome de abstinencia, síntomas extrapiramidales y sedación, debido al empleo de fármacos antipsicóticos en el tercer trimestre de gestación, en fechas cercanas al parto, por lo que es esencial la adecuada monitorización y vigilancia del recién nacido ${ }^{25}$.

Los resultados obstétricos están determinados por la enfermedad en sí misma y su sintomatología, el tratamiento médico, los factores de riesgo propios de un estilo de vida no saludable y otros problemas médicos concomitantes no reconocidos. Dada la coexistencia de factores, no se pueden atribuir ciertos resultados únicamente al empleo de psicofármacos ${ }^{26}$.

El puerperio es un período difícil, con un riesgo incrementado de deterioro psicótico ${ }^{27}$. Igualmente, las mujeres con episodios agudos en el posparto pueden tener mayor dificultad para cuidar de sus hijos ${ }^{28}$. Durante el puerperio, es importante continuar con una estrecha monitorización del estado físico y mental de la madre, el bienestar del recién nacido y el desarrollo del vínculo materno-filial a fin de detectar signos tempranos de recaída. Es beneficioso propiciar un ambiente bajo en estímulos, que favorezca la relajación y la preservación del sueño ${ }^{25}$.

El puerperio es un período difícil, con un riesgo incrementado de deterioro psicótico.

\section{Lactancia materna}

Los datos disponibles respecto al uso de antipsicóticos de primera generación en la lactancia materna indican que algunos de ellos han sido ampliamente utilizados de forma segura, como es el caso del haloperidol 29 .

En cuanto a los antipsicóticos de segunda generación, las opciones más seguras durante la lactancia materna son la olanzapina, la quetiapina y la risperidona, que se excretan en la leche materna en cantidades clínicamente no significativas. La clozapina se excreta en cantidades mayores y, además, se han documentado casos de sedación y efectos adversos hematológicos, por lo que se recomienda recurrir a fármacos alternativos con mayor experiencia de uso 29,30 .

En caso de optar por la lactancia artificial, se requiere priorizar la suspensión de la producción de leche materna con medidas no farmacológicas, pues el fármaco cabergolina disminuye los niveles de prolactina, actuando como agonista de la dopamina, lo cual es contraproducente en este tipo de pacientes ${ }^{25}$.

En todo caso, es importante observar al neonato y detectar signos de una posible intoxicación por psicofármacos (hipotonía, rigidez, somnolencia, temblor, síntomas de abstinencia) ${ }^{25}$, prestando especial atención en caso de recién nacidos de bajo peso, prematuros o que presenten cualquier otra comorbilidad ${ }^{29}$. Un recién nacido somnoliento, adormecido, hipotónico o con signos de estrés implica una deficiente lactancia, por lo que es fundamental la detección de estas situaciones y considerar otras alternativas ${ }^{29}$. Por último, estas pacientes no deben realizar el colecho con el recién nacido ${ }^{29}$.

\section{CONCLUSIONES}

La esquizofrenia es una enfermedad que causa angustia, pérdida de productividad, una menor calidad de vida y problemas médicos y mentales de carácter secundario tanto en los propios pacientes como en sus familiares ${ }^{22}$. En lo referente al tratamiento médi$\mathrm{co}$, las decisiones deben ser informadas y consensuadas con la paciente y su red de apoyo familiar ${ }^{31}$, ya que, con la información científica disponible sobre el tratamiento antipsicótico en gestantes, solo se pueden hacer recomendaciones de seguridad relativa individualizada, sopesando en cada caso la relación beneficio-riesgo ${ }^{25}$. Después del parto y durante la lactancia materna, los recién nacidos son más vulnerables, dada su exposición al tratamiento antipsicótico, y deben ser estrechamente vigilados ${ }^{25}$. 


\section{BIBLIOGRAFÍA}

1. American Psychiatric Association. Manual diagnóstico y estadístico de los trastornos mentales (DSM-5). 5. ${ }^{a}$ ed. Madrid: Editorial Médica Panamericana; 2014.

2. Barrera A. Los trastornos cognitivos de la esquizofrenia. Rev Chil Neuro-Psiquiat. 2006;44(3):215-21.

3. Brown S, Birtwistle J, Roe L, Thompson C. The unhealthy lifestyle of people with schizophrenia. Psychol Med. 1999;29(3):697-701.

4. Heald A, Pendlebury J, Anderson S, Narayan V, Guy M, Gibson $\mathrm{M}$, et al. Lifestyle factors and the metabolic syndrome in schizophrenia: a cross-sectional study. Ann Gen Psychiatry. 2017;16:12.

5. Ward M, Druss B. The epidemiology of diabetes in psychotic disorders. Lancet Psychiatry. 2015;2(5): 431-51.

6. Kerner B. Comorbid substance use disorders in schizophrenia: a latent class approach. Psychiatry Res. 2015;225(3):395-401.

7. Buckley PF, Miller BJ, Lehrer DS, Castle DJ. Psychiatric comorbidities and schizophrenia. Schizophr Bull. 2009;35(2):383-402.

8. Hor K, Taylor M. Suicide and schizophrenia: a systematic review of rates and risk factors. J Psychopharmacol. 2010;24(4 Suppl):81-90.

9. Harvey PD, Velligan DI, Bellack AS. Performancebased measures of functional skills: usefulness in clinical treatment studies. Schizophr Bull. 2007;33(5): 1138-48.

10. Thara R, Kamath S. Women and schizophrenia. Indian J Psychiatry. 2015;57(Suppl 2):S246-51.

11. Newport DJ, Calamaras MR, DeVane CL, Donovan J, Beach AJ, Winn S, et al. Atypical antipsychotic administration during late pregnancy: placental passage and obstetrical outcomes. Am J Psychiatry. 2007; 164(8):1214-20.

12. Stahl SM. Psicofarmacología esencial. Bases neurocientíficas y aplicaciones clínicas. 2. ${ }^{a}$ ed. Barcelona: Ariel Neurociencia; 2000.

13. Picchioni MM, Murray RM. Schizophrenia. BMJ. 2007; 335(7610):91-5.

14. Mendoza G, Aguilera C. Seguridad de los fármacos antipsicóticos durante el embarazo. Med Clin (Barc). 2004;122(12):475-6.

15. Robinson GE. Treatment of schizophrenia in pregnancy and postpartum. J Popul Ther Clin Pharmacol. 2012; 19(3):e380-6.

16. Babu GN, Desai G, Chandra PS. Antipsychotics in pregnancy and lactation. Indian J Psychiatry. 2015; 57(Suppl 2):S303-7.

17. Camuñas Palacín A, Grigg J, Gilbert H, Worsley R, Gavrilidis E, Kulkarni J. Seguridad de los antipsicóticos atípicos en el embarazo. Psiquiatr Biol. 2016;23(1): 23-8.

18. NICE. Antenatal and postnatal mental health: clinical management and service guidance. Clinical guideline. Londres: National Institute for Health and Care Excellence; 2014.

19. Jones I, Chandra PS, Dazzan P, Howard LM. Bipolar disorder, affective psychosis, and schizophrenia in pregnancy and the post-partum period. Lancet. 2014; 384(9956): 1789-99.

20. Seeman MV. Intervention to prevent child custody loss in mothers with schizophrenia. Schizophr Res Treatment. 2012;2012:796763.

21. Kopelowicz A, Liberman RP, Zárate R. Recent advances in social skills training for schizophrenia. Schizophr Bull. 2006;32 Suppl 1:S12-23.

22. Florit-Robles A. La rehabilitación psicosocial de pacientes con esquizofrenia crónica. Apuntes Psicol. 2006;24(1-3):223-44.

23. Judd F, Komiti A, Sheehan P, Newman L, Castle D, Everall I. Adverse obstetric and neonatal outcomes in women with severe mental illness: to what extent can they be prevented? Schizophr Res. 2014;157(1-3): 305-9.

24. Vigod SN, Kurdyak PA, Dennis CL, Gruneir A, Newman A, Seeman MV, et al. Maternal and newborn outcomes among women with schizophrenia: a retrospective population-based cohort study. BJOG. 2014;121(5): 566-74.

25. Galbally M, Snellen M, Power J. Antipsychotic drugs in pregnancy: a review of their maternal and fetal effects. Ther Adv Drug Saf. 2014;5(2):100-9.

26. Howard L, Seneviratne T. Management of women with psychosis during pregnancy. Prog Neurol Psychiatry. 2008; 12(2):9-12.

27. Seeman MV. Clinical interventions for women with schizophrenia: pregnancy. Acta Psychiatr Scand. 2013; 127(1): 12-22.

28. Vigod SN, Ross LE. Epidemiology of psychotic symptoms during pregnancy and postpartum in women with schizophrenia. 2010;6(1):17-21.

29. McAllister-Williams RH, Baldwin DS, Cantwell R, Easter A, Gilvarry E, Glover V, et al.; endorsed by the British Association for Psychopharmacology. British Association for Psychopharmacology consensus guidance on the use of psychotropic medication preconception, in pregnancy and postpartum 2017. J Psychopharmacol. 2017;31(5):519-52.

30. Kohen D. Psychotropic medication and breast-feeding. Adv Psych Treat. 2005;11(5):371-9.

31. Vogel MI, León F, Torres R, Crossley NA. Antipsicóticos de primera y segunda generación en esquizofrenia: eficacia, efectividad y efecto de la dosis utilizada. Ars Med Rev Cienc Med. 2017;42(1):41-8. 